\title{
Elogios y críticas de la próxima estructura de ponderaciones del índice de precios al consumidor en México
}

\author{
Praise for and criticism of the next weight structure \\ of the mexican consumer price index
}

\section{Carlos Guerrero de Lizardi *}

\section{Resumen}

Nuestros propósitos son los siguientes: 1) recordar que el índice de precios al consumidor es un objeto científico que está hecho para medir el poder de compra de los consumidores, y no por ejemplo el desequilibrio entre las ofertas y las demandas en los mercados de bienes y servicios de una economía; 2) señalar que la buena medición tiene fundamentos, específicamente en el caso de que nos ocupa fundamentos microeconómicos; 3) proponer algunas reflexiones metodológicas sobre el cambio de base del índice de precios al consumidor; y 4) utilizando las ENIGH 2010 y 4.2014 , ilustrar que el gasto representa otra dimensión de la desigualdad en nuestro país.

\section{Abstract}

Our purposes are as follows: 1) to point out that the consumer price index is a scientific object -that is compile to measure the purchasing power of consumers, not for example the imbalance in goods markets; 2) to point out that good measurement has fundamentals, specifically in our case microeconomic fundamentals; 3) to propose some methodological reflections on the change of base of the consumer price index; and 4) using two Expenditures Surveys illustrate that spending represents another dimension of inequality in our country.

ECONOMÍA INFORMA | 402 |ENERO-FEBRERO 2017 |
* Jefe de la División de Posgrado de la Facultad de Economía, Universidad Nacional Autónoma de México, cgdl@unam.mx.

Palabras clave

Índice de precios al consumidor, encuesta de gastos, sesgos por sustitución

Key words

Consumer Price Index, Expenditure Survey, Substitution Biases

JEL

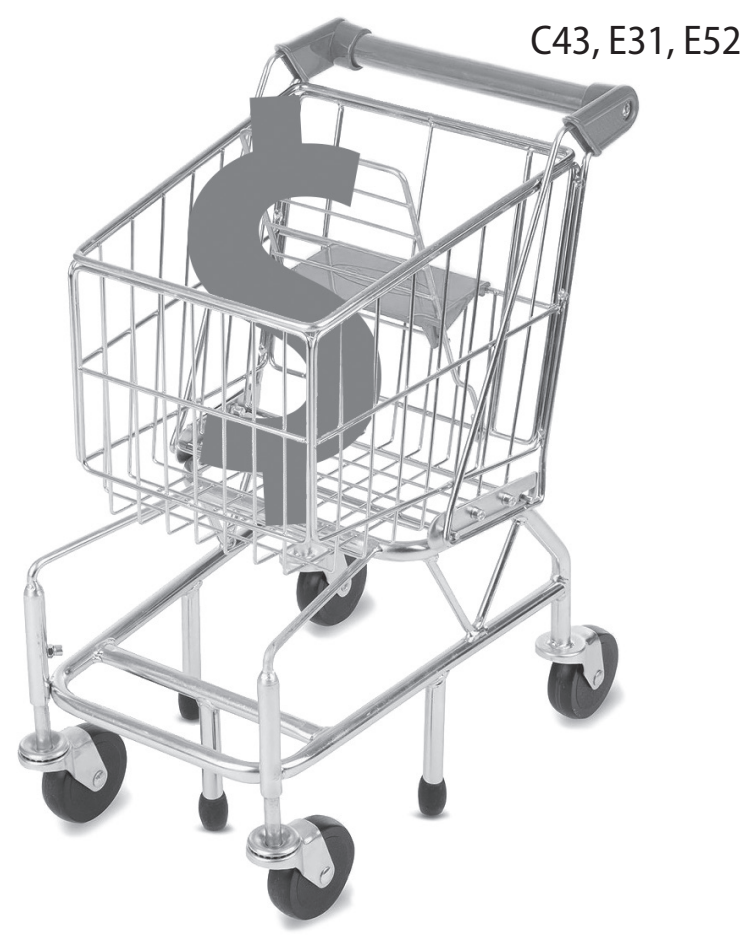




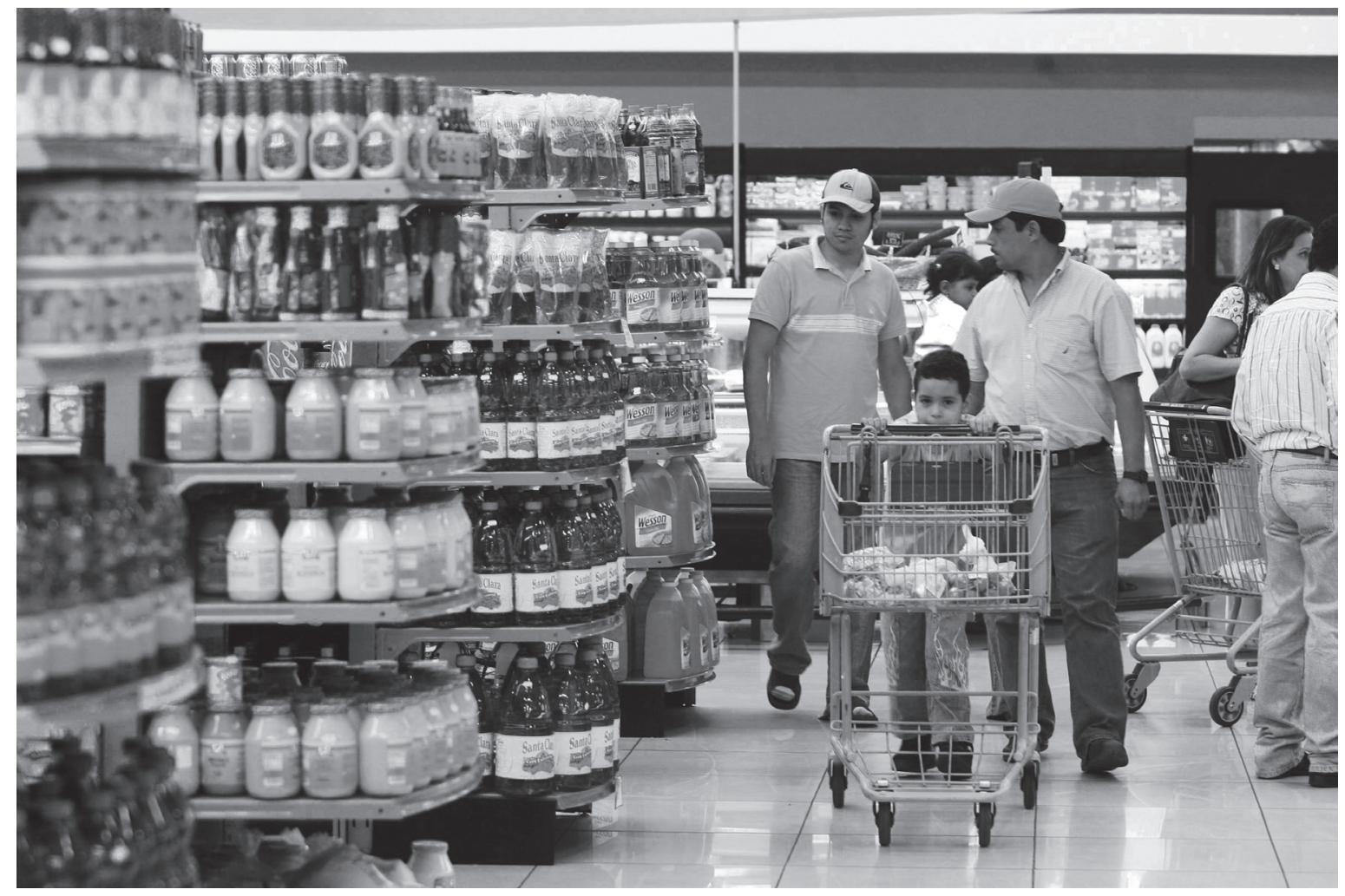

\section{Introducción}

"Observational, true, and theoretical variables; an important distinction".

Trygve Haavelmo

"Fuller utilization of the concepts and hypotheses of economic theory (in a sense described below) as a part of the processes of observation and measurement promises to be a shorter road, perhaps even the only possible road, to the understanding of cyclical fuctuations." Tjalling C. Koopmans

A raíz de la consulta pública realizada en el año 2015, la Junta de Gobierno de nuestro Instituto de Estadística anunció que a finales del 2016 realizaría un cambio de base del índice de precios al consumidor, en adelante IPC (INegi, 7 de diciembre de 2015). Específicamente, la base "segunda quincena de diciembre de 2010 " elaborada a partir de los micro- datos de la Encuesta Nacional de Ingreso y Gasto de los Hogares 2010 sería sustituida por la estructura "segunda quincena de septiembre" a partir de la Encuesta Nacional de Gasto de los Hogares del 2012 y 2013.

Los propósitos de esta nota "de elogios y críticas" son los siguientes. Inicialmente recordar que el índice de precios al consumidor es un objeto científico, en particular un estadístico, que está hecho para medir el poder de compra de los consumidores, y no por ejemplo el desequilibrio entre las ofertas y las demandas en los mercados de bienes y servicios de una economía. Posteriormente recordar que la buena medición tiene fundamentos, como se evidencia en las citas de Haavelmo (1944, p. 5) y Koopmans (1947, p. 162), específicamente en el caso de que nos ocupa fundamentos microeconómicos. Finalmente, proponer algunas reflexiones metodológicas sobre el cambio de base y realizar algunos ejercicios estadísticos 
utilizando las ENIGH 2010 y 2014, con el propósito de ilustrar que el consumo representa otra dimensión de la desigualdad en nuestro país - de la mano de muchas otras, entre otros ejemplos del ingreso, la riqueza, la educación o la salud (Filmery y Pritchett 1999, Kennickell 2007, Jantti et al. 2008, y Ruiz, 2011).

\section{2. ¡Es el poder de compra!}

Al momento el antecedente más remoto que conocemos del ipc es William Fleetwood ([1707, 1745], 1969), a decir de Balk (2008, p. 6) el genuino padre de los números índices. El Arzobispo de Ely -referenciado repetidamente en los mejores términos por Adam Smith en su "Riqueza de las Naciones" de 1776- con el propósito de estimar el poder de compra de un estudiante de Oxford entre 1460 y 1707 (Fleetwood, 1969, p. 49) construyó un índice simple compuesto por " 5 quarters of wheat, 6 yards of cloth" y, no podía ser de otra manera, "4 hogsheads of beer". ${ }^{1}$ El especialista W. Erwin Diewert (1988, p. 4) nos propone el siguiente segundo lejano antecedente:

perhaps the next independent discovery of the tabular standard was made by the Legislature of Massachusetts in 1780. An account of this discovery is given by Willard Fisher (1913). A tabular standard was used to index the pay of soldiers fighting in the Revolutionary War (a massive inflation had drastically reduced the real value of the fixed nominal pay of the soldiers). The constant quantity basket was 5 bushels of

1 Entre otras menciones del padre de la economía a Fleetwood destacamos la siguiente (1776, I.11.119): "Bishop Fleetwood and Mr. Duprè de St. Maur are the two authors who seem to have collected, with the greatest diligence and fidelity, the prices of things in ancient times. It is somewhat curious that, though their opinions are so very different, their facts, so far as they relate to the price of corn at least, should coincide so very exactly." corn, 68 and 4/7 pounds of beef, 10 pounds of sheep's wool and 16 pounds of sole leather.

El antecedente moderno es el IPC de nuestro vecino país del norte. Ante la pérdida del poder de compra de los salarios durante las primeras décadas del siglo pasado, el Bureau of Labor Statistics, en adelante BLs, estimó un índice del costo de vida para, precisamente, determinar la magnitud del incremento de los ingresos de los trabajadores. Su IPC se publicó así por primera ocasión en el año de 1921. Cabe señalar que actualmente el BLs continúa compilando el IPC americano -y no por ejemplo el Bureau of Economic Analysis, la agencia responsable de elaborar su Sistema de Cuentas Nacionales-, utilizando como insumos la información de la Consumer Expenditure Survey, y el seguimiento de los precios de los bienes y servicios determinados en su canasta.

Dicho lo anterior debe quedar claro que el objeto estadístico que nos ocupa, en primer lugar, no está ligado principalmente a la macroeconomía en general, y a la teoría y política monetarias en particular -por ejemplo, a la medición de la brecha de producto y a la determinación de la tasa de interés de referencia dada una meta inflacionaria-, sino a la estimación del poder de compra de los ingresos de las personas; y en segundo, que el dicho "su finalidad es proveer a la economía del país de moneda nacional y su objetivo prioritario es procurar la estabilidad del poder adquisitivo de dicha moneda" que aparece en el portal del Banco de México, como en otros Bancos Centrales, está erróneamente parafraseado, ya que el poder adquisitivo "de la moneda" está determinado por las decisiones del consumidor relativas a qué, cuánto, y dónde comprar. Llevado al absurdo, una unidad monetaria, una moneda de un peso mexicano, no tiene poder de compra, ¿o sí amable lector? 
Carlos Guerrero de Lizardi |

Elogios y críticas de la próxima estructura de ponderaciones

del indice de precios al consumidor en México

\section{Para resolver algunos malentendidos respecto al índice de precios al consumidor}

Para entender cabalmente qué es el IPC tenemos que recordar, en primer lugar, que estamos lidiando con un número índice de Laspeyres:

$$
P_{L}^{t}=\frac{\sum_{n=1}^{N} q_{n}^{0} p_{n}^{t}}{\sum_{n=1}^{N} q_{n}^{0} p_{n}^{0}}=\frac{q^{0} * p^{t}}{q^{0} * p^{0}}
$$

Utilizando la típica definición de precio relativo reescribimos a (1) así:

$$
\begin{gathered}
r_{N}^{t}=\frac{p_{n}^{t}}{p_{n}^{0}} \\
P_{L}^{t}=\frac{\sum_{n=1}^{N} q_{n}^{0} p_{n}^{0} r_{n}^{t}}{\sum_{n=1}^{N} q_{n}^{0} p_{n}^{0}}=\sum_{n=1}^{N} \frac{q_{n}^{0} p_{n}^{0}}{x^{0}} r_{n}^{t}=\sum_{n=1}^{N} s_{n}^{0} r_{n}^{t}
\end{gathered}
$$

La ecuación (3) evidencia por qué un índice de Laspeyres puede entenderse como una suma ponderada de precios relativos, siendo las ponderaciones la participación en el periodo base del gasto destinado a la compra de cada bien y servicio. Al respecto, el National Research Council (2002, p. 75) afirma:

this way of thinking about the price index is useful because it shows so clearly how the Laspeyres 'solves' the problem of marking a single index in a situation where the price of each good has changed in a different way. Each of the $N$ goods has its own rate of inflation, represented by its price relative. The Laspeyres averages these price relatives, each weighted according to the good's importance in the base period.

Para comprender de manera profunda qué es el IPC tenemos que recordar, en segundo lugar, que actualmente algunos Institutos fundamentan su estadístico ya sea como un índice del costo de vida (en adelante coli), destacadamente el BLS americano, o meramente como un índice del costo de una canasta de bienes (en adelante COGI), como lo hace Eurostat (Guerrero, 2014). Por su parte el INEGI no se ha atrevido a señalar explícitamente si su programa se inspira en un COGI o un COLI, pero como abundaremos más adelante su preferencia revelada es correcta en la medida que habla de "prever el sesgo por la antigüedad de los ponderadores y la canasta" (INEGI, 7 de diciembre de 2015, lámina 8).

Como argumento de autoridad respecto a la conveniencia de fundamentar al IPC como un Coli citamos un documento seminal del premio Nobel Kenneth Arrow escrito ya hace mucho tiempo (1958, pp. 77-8):

A consumers' price index is a measure for one period of time, say 1 , with respect to another period, say 0 . It is generally defined as the ratio of the expenditures needed in time 1 to maintain a given standard of living, to the expenditures needed in time 0 for the same purpose. The standard of living for this purpose clearly cannot be identified with a fixed basket of commodities... The Government statistician, for obvious reasons, shies away from the notion of trying the objective-seeming price index to the subjective concepts of utility or satisfaction, but in fact there is no escape in this proposition. Any attempt to explain the meaning of the cost-of-living index must eventually come to the notions just descri- 


\section{Gráfica 1 IPC de carne de pollo/IPC de carne de res}

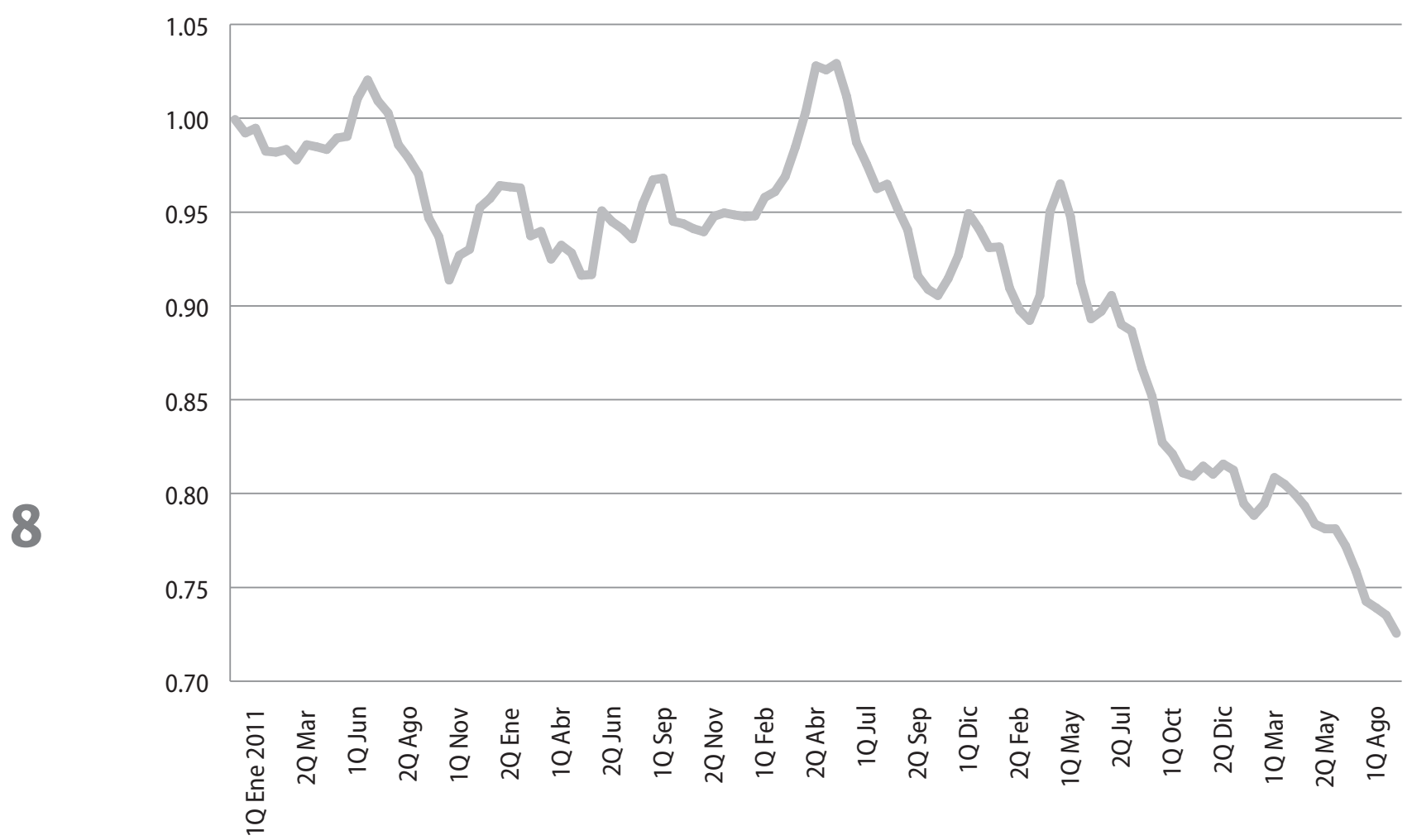

Fuente: INEGI (7 de diciembre de 2015, lámina 7).

bed unless we are to stop at banal tautologies analogous to 'national income is that which is measured by national income statisticians'.

\section{Dime en qué (y cuánto) gastas, y te diré quién eres}

En su presentación del 15 de enero-15 de febrero de 2015, los hacedores de estadísticas de nuestro país justificaron el cambio de año base del INPC así (láminas 8 y 9):

- El cálculo de la inflación no sólo depende de la representatividad de los precios involucrados, sino también de la actualidad o vigencia del resto de los componentes del indicador, como son la canasta, los ponderadores y el sistema de agregación.
- Los manuales internacionales de los Índices de Precios al Consumidor recomiendan que se realicen actualizaciones periódicas al menos cada 5 años para garantizar la representatividad del índice. También recomiendan la utilización de clasificaciones y nomenclaturas de productos y actividades reconocidas por Naciones Unidas, con el fin de permitir la comparabilidad entre los países.

- Reflejar adecuadamente la transformación de la estructura del consumo de los hogares causada por los cambios de precios relativos.

- Incorporar los cambios en las preferencias de los consumidores, que tienen su origen en la aparición en el mercado de productos y servicios novedosos, o en la desaparición de los que se han vuelto obsoletos y salen del mercado. 


\section{Carlos Guerrero de Lizardi |}

Elogios y críticas de la próxima estructura de ponderaciones

del indice de precios al consumidor en México

- Investigar el impacto de ampliar la cobertura del Î́ndice, incluyendo áreas rurales y pequeñas zonas urbanas, con una población inferior a los 15 mil habitantes, cercanas a las localidades urbanas consideradas en la muestra.

Las cinco justificaciones propuestas por el INEGI indican claramente que el IPC es un objeto científico, específicamente estadístico. Se habla de la representatividad tanto de la canasta como de los precios de los bienes y servicios que la componen, del método de agregación, y de la inclusión de localidades menores de 15000 habitantes, entre otros asuntos clave. Si bien no es nuestro objetivo abordarlos detalladamente aquí, basten algunas menciones. La representatividad está cubierta sólo parcialmente por el lado de la Encuesta Nacional de Gasto de los Hogares, pero no por el lado de la recolección de los precios, ya que se aplica un muestreo no probabilístico (INEGI, 2013, p. 34). ${ }^{2}$ El BLs americano utiliza, por ejemplo, una Point-of-Purchase Survey para determinar los lugares en los que levanta la información (Greenlees y McClelland, 2008, p. 4). El método de agregación es plutocrático. Otras opciones a la mano son el democrático o la mediana del gasto (Guerrero, 2010). Representa un par de pasos adelante la incorporación de áreas menos urbanas y el uso, por primera

2 La referencia corresponde a la metodología de la base que será sustituida pronto ya que la siguiente promesa "la metodología a aplicarse en el INPC se divulgará a partir del 24 de octubre de 2016" (INEGI, diciembre de 2015, lámina 19) no ha sido cumplida. Parece que en la próxima base se aplicará un muestreo mixto, es decir, probabilístico y no probabilístico. Amable lector, no le quepa duda, para que nuestro INEGI mejore la calidad de sus mediciones requiere lanzar más encuestas e incrementar su frecuencia, lo que implica que la sociedad mexicana debe estar dispuesta a incrementar sustancialmente su presupuesto. ocasión, de regresiones hedónicas para ajustar por calidad algunos índices de precios. Como puntos de referencia de nuestra región recordemos que el IPC americano es netamente urbano, pero el canadiense es urbano y rural.

Para exponer el cambio de preferencias del consumidor en el tiempo, nuestro Instituto de Estadística propuso el siguiente ejemplo (7 de diciembre de 2015, lámina 7):

Tabla 1

Cambio de preferencias por precios relativos

\begin{tabular}{lccc}
\hline \multicolumn{1}{c}{ Genérico } & $\begin{array}{c}\text { Ponderador } \\
\text { vigente }\end{array}$ & $\begin{array}{c}\text { Ponderador } \\
\text { Con ENGASTO } \\
\text { 2012-2013 }\end{array}$ & $\begin{array}{c}\text { Variación } \\
\text { porcentual }\end{array}$ \\
\hline $\begin{array}{l}\text { Carne de } \\
\text { pollo }\end{array}$ & 1.3230 & 1.3345 & 0.87 \\
Carne de res & 1.7762 & 1.6810 & -5.36 \\
\hline
\end{tabular}

Fuente: INEGI (7 de diciembre de 2015, lámina 7).

Los agentes económicos respondemos a las variaciones de los precios relativos, por lo que si la estructura de ponderaciones no se actualiza frecuentemente -Francia, Reino Unido y Suecia lo hacen cada año, y nuestros socios norteamericanos, ambos, cada dos ańos- el índice de precios contiene sesgos por sustitución de bienes y servicios, y por la desaparición de productos y la introducción de otros, entre otros errores y sesgos muestrales y no muestrales de medición ampliamente documentados en la literatura especializada (Guerrero, 2017). Así por ejemplo, en la gráfica y cuadros anteriores se observa un abaratamiento relativo de la carne de pollo respecto a la de res, y la reacción de los consumidores se evidencia en el cambio de sus ponderadores, esto es, en el incremento de uno $(+0.87 \%)$, y la disminución del otro $(-5.36 \%)$. Por tanto, el no cambio de base está introduciendo un sesgo por sustitución que daña la medición de los precios al consumidor actualmente. Querido lector, ahora ima- 
Tabla 3

Ingreso por decil según la ENIGH 2014, en pesos constantes del 2014

\begin{tabular}{lcccccccccc}
\hline Decil & 1 & 2 & 3 & 4 & 5 & 6 & 7 & 8 & 9 & 10 \\
\hline Ingreso & 6,902 & 12,035 & 16,058 & 20,082 & 24,439 & 29,532 & 36,094 & 45,593 & 62,840 & 143,850 \\
\hline
\end{tabular}

Fuente: Tabulados básicos de la ENIGH 2014.

gine los efectos de algo similar derivado de las variaciones de los precios relativos de los ¡283 genéricos del IPC vigente!

El cambio de base está fundamentado en la información de la Encuesta Nacional de Gasto de los Hogares del 2012 y 2013 (ENGASTO en adelante). Es nuestra obligación mencionar que esta suma aritmética de gastos de dos encuestas complejas - para determinar los ponderadores- si bien tiene algún sentido práctico, estadísticamente es una operación absolutamente incorrecta. Creemos que lo anterior puede constituir una explicación, entre otras, del retraso en la publicación de la nueva estructura del IPC y de su metodología.

Para abrir boca la siguiente tabla contiene la distribución del gasto en dos ejercicios estadísticos de nuestro Instituto de Estadística.

Tabla 2

Distribución porcentual del gasto por quintiles

\begin{tabular}{lccccc}
\hline $\begin{array}{c}\text { Encuesta/ } \\
\text { Quintiles }\end{array}$ & 1 & 2 & 3 & 4 & 5 \\
\hline ENGASTO 2013 & $4.96 \%$ & $9.52 \%$ & $14.27 \%$ & $21.58 \%$ & $49.67 \%$ \\
ENIGH 2014 & $7.18 \%$ & $11.40 \%$ & $14.95 \%$ & $20.67 \%$ & $45.80 \%$ \\
\hline
\end{tabular}

Fuente: Tabulados básicos de la ENGASTO 2013 y ENIGH 2014.

El contenido de la tabla anterior nos recuerda la desigual distribución del gasto en nuestro país. Y es que la desigualdad tiene, entre otras, tres dimensiones, la de los ingresos, la de los gastos, y la de la riqueza. Digamos que una quinta parte de los hogares mexicanos ejerce la mitad del gasto total; o puesto con otras palabras, por cada peso que gastan las familias más pobres, relativamente hablando, las fami- lias más ricas gastan diez pesos. En este sentido se espera que el tamaño del ponderador del grueso de los códigos correspondientes a los deciles más ricos, relativamente hablando, sea próximo a uno. Puesto coloquialmente, la rebanada del pastel que toman los deciles más privilegiados, de cada uno de los códigos de gasto, es enorme, comparada a la minúscula rebanada que toma el resto de la sociedad. Y para que usted, estudiante y profesor, ubique la posición relativa de su hogar, la siguiente tabla contiene el ingreso promedio por decil.

La siguiente tabla (tabla 4)contiene alguna información respecto a la proporción del gasto ejercido por decil en algunos rubros seleccionados. A excepción del gasto en huevo, el resto de los rubros de gasto evidencia una terrible desigualdad. Por ejemplo, el decil más rico ejerce el $85 \%$ del gasto ejercicio en la compra de un seguro médico. Y la salud es clave para entender el devenir histórico de una sociedad, escribió recientemente el Nobel A. Deaton.

\section{Reflexiones finales}

Si bien durante la primera mitad del siglo pasado mentes brillantes de nuestra ciencia se dedicaron a diseñar lo que hoy conocemos como Sistema de Cuentas Nacionales, pero actualmente los economistas nos volvimos más bien usuarios de las estadísticas. En este sentido, la literatura reconoce que tenemos que dedicar más tiempo a la genuina comprensión de los datos. Esperamos que esta nota se entienda como una invitación al respecto. 


\section{Carlos Guerrero de Lizardi |}

Elogios y críticas de la próxima estructura de ponderaciones

del indice de precios al consumidor en México

Tabla 4

Distribución del gasto por decil según las ENIGH 2010 y 2014, en por ciento

\begin{tabular}{|c|c|c|c|c|c|c|c|c|c|c|c|}
\hline \multirow{2}{*}{ Rubros del gasto } & \multirow{2}{*}{ Años } & \multicolumn{10}{|c|}{ Deciles } \\
\hline & & I & II & III & IV & V & VI & VII & VIII & IX & $x$ \\
\hline \multirow{2}{*}{ Gasto corriente monetario } & 2010 & 3.2 & 4.1 & 5.2 & 6.0 & 7.0 & 8.2 & 9.8 & 11.8 & 15.6 & 29.2 \\
\hline & 2014 & 3.0 & 4.2 & 5.1 & 6.3 & 7.0 & 7.9 & 9.3 & 11.4 & 15.0 & 30.8 \\
\hline \multirow{2}{*}{ Alimentos, bebidas y tabaco } & 2010 & 4.7 & 5.9 & 7.1 & 7.7 & 8.5 & 9.7 & 10.8 & 12.1 & 14.0 & 19.6 \\
\hline & 2014 & 4.4 & 5.9 & 6.9 & 7.9 & 8.6 & 9.4 & 10.4 & 12.2 & 14.1 & 20.3 \\
\hline \multirow{2}{*}{ Carnes } & 2010 & 3.9 & 6.0 & 7.6 & 8.4 & 9.4 & 10.8 & 11.7 & 12.8 & 14.0 & 15.2 \\
\hline & 2014 & 3.7 & 5.5 & 7.2 & 8.3 & 9.6 & 10.2 & 11.3 & 13.2 & 14.4 & 16.7 \\
\hline \multirow{2}{*}{ Pescados y mariscos } & 2010 & 3.6 & 5.0 & 6.1 & 5.8 & 7.8 & 8.9 & 9.3 & 11.1 & 16.2 & 26.3 \\
\hline & 2014 & 3.8 & 4.9 & 6.1 & 6.8 & 7.3 & 7.9 & 10.9 & 11.2 & 14.2 & 27.0 \\
\hline \multirow{2}{*}{ Huevo } & 2010 & 8.4 & 9.9 & 10.2 & 10.7 & 10.7 & 10.8 & 10.7 & 10.1 & 9.7 & 8.8 \\
\hline & 2014 & 7.4 & 9.1 & 9.8 & 10.9 & 10.8 & 10.8 & 10.7 & 11.3 & 9.8 & 9.2 \\
\hline \multirow{2}{*}{ Frutas } & 2010 & 3.5 & 4.4 & 6.1 & 6.5 & 8.5 & 8.7 & 11.3 & 11.5 & 15.0 & 24.4 \\
\hline & 2014 & 3.1 & 5.0 & 6.0 & 7.2 & 7.9 & 9.3 & 9.9 & 12.7 & 14.4 & 24.5 \\
\hline \multirow{2}{*}{ Tabaco } & 2010 & 5.8 & 4.7 & 4.5 & 8.8 & 6.5 & 7.9 & 12.7 & 12.1 & 17.4 & 19.7 \\
\hline & 2014 & 4.0 & 5.2 & 4.9 & 5.0 & 6.8 & 5.9 & 14.4 & 13.2 & 16.6 & 24.0 \\
\hline \multirow{2}{*}{ Agua } & 2010 & 3.4 & 5.1 & 6.8 & 8.2 & 8.2 & 9.5 & 10.9 & 12.5 & 14.2 & 21.3 \\
\hline & 2014 & 3.8 & 4.8 & 5.9 & 8.0 & 8.6 & 10.1 & 11.1 & 12.6 & 15.1 & 20.0 \\
\hline \multirow{2}{*}{ Electricidad y combustibles } & 2010 & 4.2 & 5.7 & 6.7 & 7.6 & 8.4 & 9.4 & 10.7 & 12.0 & 14.0 & 21.3 \\
\hline & 2014 & 3.9 & 5.7 & 6.7 & 7.8 & 8.4 & 9.3 & 10.4 & 11.8 & 14.1 & 21.9 \\
\hline \multirow{2}{*}{ Servicios médicos } & 2010 & 3.3 & 3.5 & 4.3 & 4.5 & 5.5 & 6.3 & 7.4 & 10.9 & 16.7 & 37.6 \\
\hline & 2014 & 2.5 & 5.6 & 4.2 & 6.4 & 6.3 & 6.1 & 7.3 & 9.8 & 14.2 & 37.5 \\
\hline \multirow{2}{*}{ Medicamentos recetados } & 2010 & 4.7 & 5.3 & 5.6 & 5.4 & 7.3 & 7.3 & 8.1 & 9.9 & 13.9 & 32.6 \\
\hline & 2014 & 4.1 & 6.1 & 6.0 & 8.4 & 6.5 & 8.4 & 8.6 & 10.6 & 14.0 & 27.4 \\
\hline \multirow{2}{*}{ Seguro médico } & 2010 & 0.1 & 0.1 & 0.2 & 2.0 & 2.4 & 0.9 & 2.1 & 4.8 & 2.8 & 84.6 \\
\hline & 2014 & 0.2 & 0.3 & 0.0 & 0.1 & 0.3 & 0.5 & 7.1 & 2.2 & 11.0 & 78.3 \\
\hline \multirow{2}{*}{ Adquisición de vehículos de uso particular } & 2010 & 1.0 & 1.7 & 1.5 & 1.7 & 4.4 & 2.5 & 5.3 & 8.8 & 22.2 & 50.8 \\
\hline & 2014 & 0.8 & 0.6 & 1.1 & 2.2 & 2.5 & 3.1 & 6.8 & 6.7 & 18.9 & 57.3 \\
\hline
\end{tabular}

Fuente: Tabulados básicos de las ENIGH 2010 y 2014. 


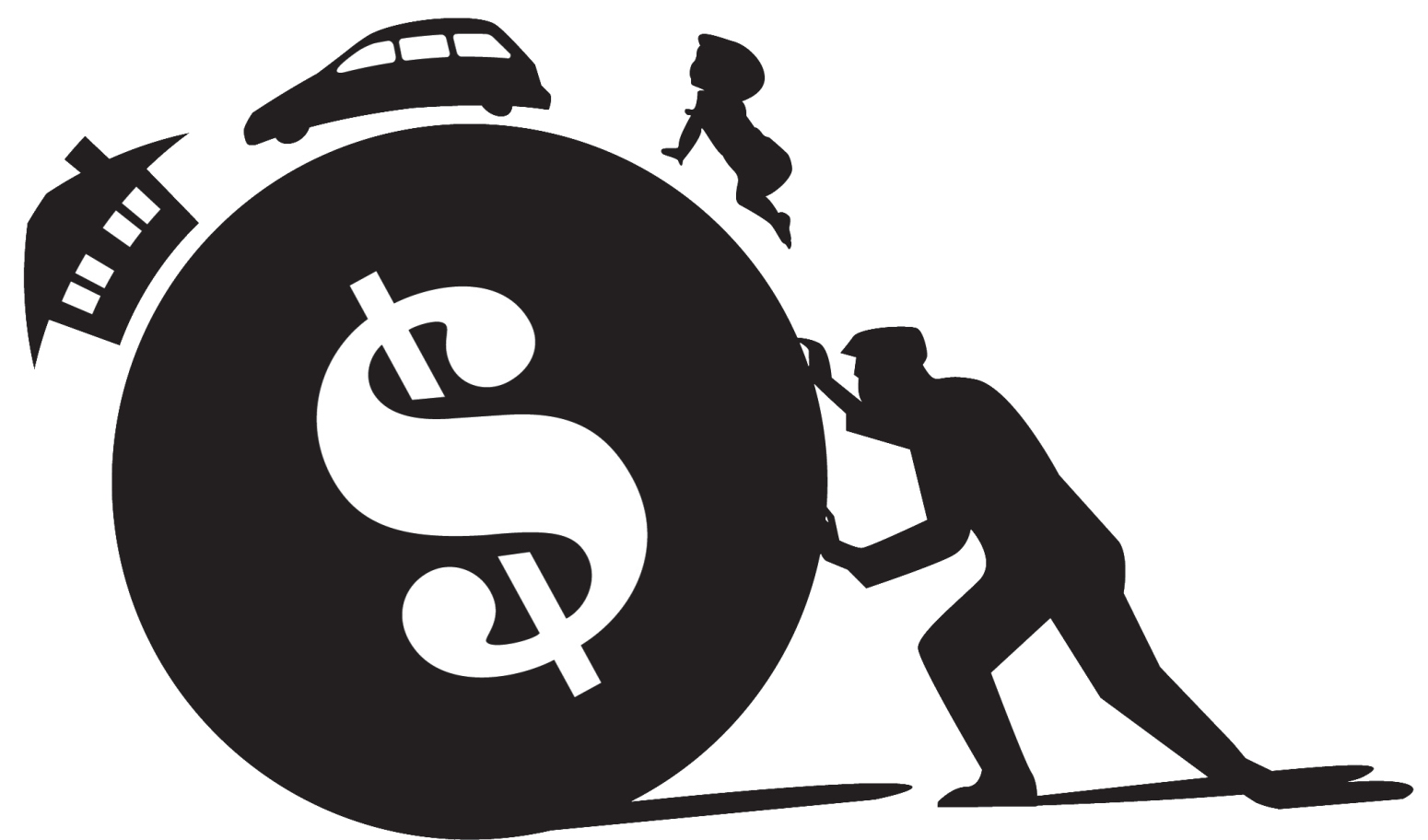

\section{Referencias}

Arrow, K. J. (1958). "The measurement of price changes", The Relationship of Prices to Economic Stability and Growth, Joint Economic Committee U. S. Congress, U. S. Government Printing Office, pp. 77-87.

Balk, B. M. (2008). Price and Quantity Index Numbers: Models for Measuring Aggregate Change and Difference, Cambridge University Press.

Diewert, W. E. (1988). "The early history of price index research", NBER Working Paper Series, No. 2713.

Filmer, D. y L. Pritchett (1999). “The effect of household wealth on educational attainment: evidence from 35 countries", Population and Development Review, 25(1), pp. 85-120.

Fleetwood, W. ([1707, 1745], 1969), Chronicon Preciosum, or, an Account of English Money, the Price of Corn, and Other Commodities for the last 600 years: in a Letter to a Student in the University of Oxford, reprinted, Augustus M. Kelley Publishers.

Guerrero, C. (2010). "Alternative consumer price indexes for Mexico", Center for International Development Working Paper, No. 42, John F. Kennedy School of Government, Harvard University.

Guerrero, C. (2014). “A case of 'No man's land' in economics: the theory of price indexes and its applications", International Journal of Pluralism and Economics Education, 5: 2, 144-56.

Guerrero, C. (2017). "Concerns derived from a closer look at the Consumer Price Index estimation", por aparecer en Reality, Data and Space: International Journal of Statistics and Geography,

Haavelmo, T. (1944). "The probability approach in econometrics”, Econometrica, vol. 12, supplement, pp. iii-vi + 1-115.

INEGI, (15 de enero - 15 de febrero de 2015). "Metodología en consulta. Cambio de año base del INPC".

INEGI, (7 de diciembre de 2015). "Cambio de base del INPC 2da quincena de septiembre 2016”, en http:// www.inegi.org.mx/est/contenidos/proyectos/inp/default.aspx.

Jantti, M., E. Sierminska y T. Smeeding (2008). "The joint distribution of household income and wealth: evidence from the Luxemburg Wealth Study", OECD Social, Employment and Migration Working Papers, DELSA/ELSA/WD/SEM(2008)2.

Kennickell, A. B. (2007). "What's the difference? Evidence on the distribution of wealth, health, life expectancy and health insurance coverage", Federal Reserve Board.

Koopmans, T. C. (1947). "Measurement without theory", The Review of Economics and Statistics, 29:3, pp. 161-72.

Ruiz, N. (2011). "Measuring the joint distribution of household's income, consumption and wealth using nested Atkinson measures", Statistics Directorate Working Paper No. 20, oECD.

Smith, A. (1776). "An Inquiry into the Nature and Causes of the Wealth of Nations", en http://www. econlib.org/library/Smith/smWN.html. 